\title{
Vorwort zum Supplementband 2012
}

Online publiziert: 13. Oktober 2012

(C) Springer-Verlag Wien 2012

Als amtierende Präsidentin der der Österreichischen Gesellschaft für Tropenmedizin und Parasitologie möchte ich die Tradition fortsetzen und die vergangene Jahrestagung 2011, deren Beiträge Sie in diesem Band z. T. als Publikationen vor sich liegen haben, noch einmal kurz Revue passieren lassen.

Die 45. Jahrestagung der ÖGTP fand unter der Präsidentschaft von Univ.-Prof. Dr. Ursula WiedermannSchmidt vom 17.-19. November 2011 im Billrothaus der Gesellschaft der Ärzte in Wien statt. Im Vorfeld der Tagung fand ein Symposium zum Thema „Migration: Epidemiologie und medizinische Aspekte" statt. Das Motto der anschließenden Tagung war „From Bugs to Drugs“. Die Schwerpunktthemen umspannten die Komparative Medizin mit einem Plenarvortrag von Prof. Jensen-Jarolim vom Messerli-Institut der Veterinärmedizinischen Universität Wien und zwei eingeladenen Vorträgen von Profs. Müller und Saalmüller, ebenfalls von der Veterinärmedizinischen Universität Wien, zum Thema Biomodelle und vergleichende Immunologie.

In den folgenden Sektionen wurde ein bunter Reigen wissenschaftlicher Forschungsergebnisse präsentiert, die den Bogen von den Grundlagen der Wirt-ParasitInteraktionen und Molekularparasitologie über die Diagnostik und Epidemiologie bis hin zur klinischen Parasitologie, Vakzinologie, Tropen-, Reise- und Migrationsmedizin spannten. Am letzten Tag der Tagung fand in altbewährter Manier die Fortbildung für Ärzte mit „Neuem aus dem Impfwesen und der Reisemedizin“ statt.

Die Tagung der ÖGTP umfasst nicht nur das breite Gebiet der grundlagenorientierten und klinisch-angewandten Forschung, sie stellt auch eine Gelegenheit für das Zusammentreffen junger NachwuchsforscherInnen mit arrivierten WissenschafterInnen dar und ermöglicht den formalen wie auch den informellen Austausch von Ansichten und Ideen, der in der Forschung so wichtig ist.

Um die Leistungen unseres wissenschaftlichen Nachwuchses gebührend zu würdigen, lobt die ÖGTP anlässlich ihrer Jahrestagung stets einen Junior Award und einen Junior Poster Award aus, die von der Firma Pfizer gesponsert wurden.
Die Preisträger des Jahres 2011 waren Sarah SCHLOSSER und Coautoren (Identification of potential thioredoxin target proteins in Entamoeba histolytica using Trx-affinity chromatography; 1. Platz Junior Award), Lorenz AUER-HACKENBERG und Kollegen (Automated erythrocytapheresis in five severe cases of Plasmodium falciparum malaria in Vienna; 2. Platz Junior Award), Mirjana DRINIC und Coautoren (Pentamycin shows high anti-protozoal activity in vitro; 1. Platz Junior Poster Award), Johnnie AKGÜN und Mitautoren (Parasitic infection and their impact on vaccine responses; 2 . Platz Junior Poster Award) und Stefan MILLER (Ebola in Uganda - Medical Anthropological Aspects; 3. Platz Junior Poster Award).

Einiges dessen, was bei der Tagung vorgetragen wurde, hat nach Durchlaufen des Begutachtungsprozesses Eingang in diesen Band gefunden. Es handelt sich dabei um einen Übersichtsartikel zu Medizin und Diversitätskompetenz, fünf Originalartikel, einen Fallbericht und einen Kurzbericht, und auch in den Themen findet sich die breite Palette der Grundlagen und der klinischen Forschung wieder. Ich danke den AutorInnen für ihre Beiträge.

Großer Dank gilt auch dem Vorstand der ÖGTP und den vielen Helfern aus den Reihen der Gesellschaft, die jedes Jahr die Mühe auf sich nehmen, die örtliche Tagungsorganisation mit Erfahrung, Know-How und Tatkraft zu unterstützen. Ohne die vielen freiwilligen Hände und Köpfe wäre es sicher unmöglich, eine solche Veranstaltung durchzuführen. Herrn Mag. Christoph Hörweg, dem Schriftführer der ÖGTP, gilt dabei ganz besonderer Dank für sein Engagement.

Da trotz der Vielzahl freiwilliger Helfer eine Tagung auch Geld kostet, danke ich an dieser Stelle auch nochmals ausdrücklich allen Sponsoren, die bei der Ausrichtung der Tagung und der Erstellung dieses Bandes finanzielle Unterstützung gewährt haben, und zwar (in alphabetischer Reihenfolge) Alere $\mathrm{GmbH}$, Baxter Healthcare GmbH, Bayer Austria Ges.m.b.H. (Animal Health \& Bayer Healthcare Pharmaceuticals), GlaxoSmithKline Pharma GmbH, Medizinische Universität Wien, Merck Sharp \& Dohme Ges.m.b.H., Naturhistorisches Museum 
Wien, Nikon GmbH Vienna, Pfizer Corporation Austria $\mathrm{GmbH}$, Roche Austria GmbH, Sanofi Pasteur MSD $\mathrm{GmbH}$, sigma-tau Arzneimittel $\mathrm{GmbH}$ und Veterinärmedizinische Universität Wien.

Dieser Band wäre nicht zustande gekommen ohne den Einsatz von Frau Doz. Julia Walochnik, die als Chefredakteurin der ÖGTP alle Beiträge sammelt, sichtet, begutachten lässt und schließlich für die Veröffentlichung zusammenstellt - eine Aufgabe, die gar nicht hoch genug geschätzt werden kann!
Ich hoffe, dass Sie die Beiträge des diesjährigen Bandes wieder mit Vergnügen lesen werden und verbleibe,

Mit herzlichen Grüßen,

Ihre Anja Joachim

Präsidentin der ÖGTP 2011-2013

\section{Interessenskonflikt}

Es besteht kein Interessenskonflikt. 